\title{
Genetic Diversity and Population Structure of Traditional Chinese Herb "Chai-Hu" Resources Using Genome-Wide SNPs Through Genotyping-By-Sequencing
}

\author{
Ming Jiang ( $\sim$ jiangming314220@126.com ) \\ Heilongjiang University of Chinese Medicine

\section{Song Yan} \\ Heilongjiang University of Chinese Medicine

\section{Weichao Ren} \\ Heilongjiang University of Chinese Medicine

\section{Nannan Xing} \\ Heilongjiang University of Chinese Medicine

\section{Hongyuan Li} \\ Heilongjiang University of Chinese Medicine

\section{Meiqi Zhang} \\ Northeast Forestry University

\section{Meiqi Liu} \\ Heilongjiang University of Chinese Medicine \\ Wei Ma \\ Heilongjiang University of Chinese Medicine
}

\section{Research Article}

Keywords: Bupleurum chinense, Genetic diversity, Population structure, Cluster analysis, Genotyping-by-sequencing (GBS)

Posted Date: January 12th, 2022

DOI: https://doi.org/10.21203/rs.3.rs-1194484/v1

License: (c) (i) This work is licensed under a Creative Commons Attribution 4.0 International License. Read Full License 


\section{Abstract}

Bupleurum (named "Chai-hu") is an important traditional Chinese medicine resource in China. It has been widely used since ancient times and has antipyretic, analgesic and cholagogic functions, but there is little research on its genetic diversity. In this study, genotyping-by-sequencing (GBS) was used to detect SNP loci in 39 Bupleurum germplasm resources from different regions in China and analyse their genetic diversity. A total of $25.1 \mathrm{~Gb}$ of data was obtained by sequencing, with an average of $0.64 \mathrm{~Gb}$ per sample. After screening, 83898 high-quality SNPs were obtained. The results of genetic research were obtained by phylogenetic tree, principal component analysis and population structure analysis, and the 39 experimental materials were divided into three groups. The average observed heterozygosity and expected heterozygosity of Bupleurum populations were 0.24 and 0.17 , respectively, indicating that Bupleurum populations from five different provinces had a low level of genetic diversity. Population nucleotide diversity analysis and analysis of molecular variance showed that the percentage of intrapopulation variation was $120.88 \%$, while the percentage of interpopulation variation was only $2.46 \%$. There was relative aggregation of Bupleurum samples with the same geographical origin, but the division of population structure was not completely correlated with sample origin. The results showed that the genetic diversity of the materials was low and that the genetic variation was narrow. This provides a good basis for the genetic breeding and protection of species diversity of Bupleurum.

\section{Introduction}

Bupleurum chinense DC. is one of the most commonly used medicinal herbs belonging to the genus Bupleurum and is named "Chai-hu" (Radix Bupleuri). It has been broadly used for approximately 2000 years, with records in the historical Chinese book "The Herbal Classic of Shen Nong" [1]. B. chinense possesses diverse TCM value, including in the treatment of influenza, fever, hepatitis, pancreatitis, inflammation, malaria and depression [2, 3]. Extracts from the roots of $B$. chinense contain large amounts of bioactive flavonoids, saikosaponins (A, B, D, F), sesquiterpenes, alkaloid glycosides, saponins and volatile oils, which exhibit anti-inflammatory, antioxidant, antidepressant, antiapoptotic, and antiaddictive pharmacological functions [4-10].

As described in the Pharmacopoeia of the People's Republic of China, B. chinense is derived from the roots of Bupleurum chinense DC. (called 'Bei Chaihu') and Bupleurum scorzonerifolium Willd. (called 'Nan Chaihu') of the Umbelliferae [11]. Recently, more than 20 other counterfeit plants have been used to treat related diseases. Parts of other species have been used under same name of 'Chaihu', including Dianthus superbus L., Bupleurum pyramidalis., Bupleurum longiradiatum Turez., Stellaria dichotoma L. var. lanceolata Bge., Bupleurum smithii Wolff var. parvifolium Shan et Y. Li and other species [12]. However, research shows that different species markedly influence the pharmacological effects and medicinal value of these herbal products. For this reason, the evaluation and utilization of the genetic resources of this important herb is of great significance for future breeding. The genetic background of Bupleurum chinense DC. has typically been determined based on inter simple sequence repeat (ISSR) and simple sequence repeat (SSR) molecular markers [13-14]. However, these two kinds of DNA markers have limitations, such as a low reproducibility and limited quantity [15].

Genotyping-by-sequencing (GBS) is a common simplified genome sequencing technology that is suitable for population research, germplasm identification, genetic improvement and trait mapping in a variety of sources. This method can generate high-density and low-cost genotyping data and is appropriate for assessing the genetic variation of organisms with very large genomes. The lack of a need for a reference genome makes it very useful in nonmodel plants [16-18]. For example, the genetic diversity and population structure of 103 popcorn germplasm samples were analysed by GBS, and the genetic diversity, population structure and heterosis patterns were revealed, which provided a basis for cross breeding with existing maize germplasm resources [19]. GBS analysis of 610 pumpkin germplasm resources from global sources revealed 2071 high-quality single nucleotide polymorphisms (SNPs) distributed on 20 chromosomes. The genetic structure of different germplasm resources worldwide was studied based on this valuable information, and new gene banks were established [20]. A total of 180 common wheat populations from Asia and Europe between $30^{\circ}$ and $45^{\circ}$ latitude were

Page 2/12 
analysed using GBS, and 24767 high-quality SNPs were used to analyse their genetic diversity and population structure. The results can not only help breeders understand the genetic diversity of wheat germplasm resources but also provide valuable information for wheat genetic improvement through the exploitation of new genetic variations in this region [21].

In this study, GBS was used for the first time to analyse the genetic diversity and population structure of 39 samples of $B$. chinensis collected from different provinces of China, providing a theoretical basis for the efficient utilization and genetic breeding of $B$. chinensis germplasm resources.

\section{Materials And Methods}

\subsection{Plant materials and DNA extraction}

Thirty-nine test materials of $B$. chinensis were collected from 14 various habitats of 5 different provinces, and these samples were checked by Professor Dezhi Ma (Table 1). Fresh leaves of these plants were collected for grinding, and DNA samples were extracted using a spin column reagent (Tiangen Biotech, Beijing, China) according to the isolation kit instructions and used for amplification. DNA purity and integrity were analysed by agarose gel electrophoresis, nanodrop detection (OD260/280 ratio) and Qubit accurate quantification methods. High-quality DNA after concentration and purity determination was used for GBS library construction and sequencing. 
Table 1

Summary of the tested Bupleurum samples

\begin{tabular}{|c|c|c|c|c|c|}
\hline Species & No. & $\begin{array}{l}\text { Collection location } \\
\text { (Longitude and Latitude) }\end{array}$ & Species & No. & $\begin{array}{l}\text { Collection location } \\
\text { (Longitude and Latitude) }\end{array}$ \\
\hline \multirow{20}{*}{$\begin{array}{l}\text { B. } \\
\text { chinense } \\
\text { DC. }\end{array}$} & HB1 & Hebei(117.298;40.908) & B. chinense DC. & HRB2 & Heilongjiang(126.649;45.924) \\
\hline & HB2 & Hebei(117.275;40.869) & & HRB3 & Heilongjiang(126.906;45.902) \\
\hline & HB3 & Hebei(117.310;40.856) & & DQ1 & Heilongjiang(125.102;46.521) \\
\hline & HB4 & Hebei(117.737;41.355) & & DQ2 & Heilongjiang(125.150;46.443) \\
\hline & HB5 & Hebei(117.774;41.354) & & DQ3 & Heilongjiang(125.085;46.462) \\
\hline & HB6 & Hebei(117.774;41.297) & & ZZ1 & Heilongjiang(125.289;45.707) \\
\hline & BJ2 & Beijing $(116.789 ; 40.252)$ & & ZZ2 & Heilongjiang(125.307;45.699) \\
\hline & BJ3 & Beijing(116.858;40.427) & & ZZ3 & Heilongjiang(125.243;45.695) \\
\hline & BJ4 & Beijing(116.774;40.438) & & DF4 & Shaanxi(110.336;33.686) \\
\hline & BJ5 & Beijing(116.556;40.345) & & DF5 & Shaanxi(110.340;33.654) \\
\hline & BJ7 & Beijing(116.571;40.329) & & DF6 & Shaanxi(10.245;33.704) \\
\hline & GS1 & Gansu(105.036;33.387) & $\begin{array}{l}\text { B. } \\
\text { scorzonerifolium Willd. }\end{array}$ & MH1 & Heilongjiang(129.699;44.619) \\
\hline & GS2 & Gansu(105.007;33.374) & & MH3 & Heilongjiang(129.620;44.653) \\
\hline & GS3 & Gansu(104.912;33.386) & B. sibiricum Vest. & Q1 & Heilongjiang(129.699;44.619) \\
\hline & GS4 & Gansu(104.331;33.765) & & Q2 & Heilongjiang(129.620;44.655) \\
\hline & GS6 & Gansu(104.353;33.769) & & Q3 & Heilongjiang(129.571;44.641) \\
\hline & BQ1 & Heilongjiang $(126.129 ; 47.611)$ & $\begin{array}{l}\text { B. Iongiradiatum } \\
\text { Turcz. }\end{array}$ & DY1 & Heilongjiang(129.692;44.622) \\
\hline & BQ2 & Heilongjiang(126.092;47.587) & & DY2 & Heilongjiang(129.595;44.631) \\
\hline & BQ3 & Heilongjiang(126.068;47.585) & & DY3 & Heilongjiang(129.494;44.625) \\
\hline & $\begin{array}{l}\text { HRB1 } \\
\text { B2B1 }\end{array}$ & Heilongjiang(126.476;45.936) & & & \\
\hline
\end{tabular}

\subsection{GBS library construction and data analysis}

The GBS library was constructed according to a procedure described earlier with slight revisions [22-24]. Briefly, total genomic DNA was diluted to $20 \mathrm{ng} / \mu \mathrm{l}$ with distilled water for the preparation for GBS analysis, and was digested with Mse] (New England Biolabs) at $80{ }^{\circ} \mathrm{C}$ for $30 \mathrm{~min}$. The digested DNAs were ligated with barcoded adapters using T4 ligase, and all ligated fragments and PCR products were cleaned up using a QIAquick PCR purification kit (Qiagen). The cleaned PCR products were quantified, and the correct-sized fragments were selected with an E-gel system. The library concentration was estimated by a Qubit 2.0 fluorometer, and the library was sequenced on an Illumina HiSeq PE150 (Illumina). Then, clean reads were aligned to the simulated reference genome after analysis of clustering with stacks software. According to the suitable mapped reads from 39 Bupleurum samples, SNPs were detected and genotyped using SAMTOOLS software.

\subsection{Population genetic structure analysis}


The pedigree relationship of Bupleurum resources was determined using the unsupervised clustering method with ADMIXTURE software [25]. The admixture model was run five independent times with $\mathrm{K}$ values ranging from 2 to 11 . The individual samples were designated to each cluster after characterization of the number of $\mathrm{K}$ values according to the optimal grouping.

Evolution analysis was checked with TreeBest software (http://treesoft.sourceforge.net/treebest.shtml)using the NJ (neighbour-joining) method with 1,000 bootstrap values and shown with a phylogenetic tree. Principal component analysis (PCA) was performed with GCTA software (http://cnsgenomics.com/software/gcta/pca.html), and several components were analysed by a covariance matrix. We used the first three components with high eigenvalues for further analysis, and the results of PCA were visualized using $\mathrm{R}$.

\subsection{Genetic diversity analysis}

Arlequin software [26] was used to calculate the observed heterozygosity $(\mathrm{Ho})$ and expected heterozygosity $(\mathrm{He})$. Ho is shown as the ratio of the number of heterozygous individuals to the total number of samples. He was determined according to Nei's method [27], which describes the average distance between samples within the same subpopulation. Nucleotide diversity $(\pi)$ was also calculated to analyse the subpopulation-level polymorphism, and AMOVA revealed the genetic variation among the different subpopulations based on the filtered SNP loci.

\section{Results}

\subsection{GBS analysis and SNP characterization}

For the 39 Bupleurum accessions, GBS sequencing produced 1.3 billion clean reads, and more than 1.9 million reads per sample were retained for alignment. The number of clean reads ranged from 1,961,510 (HB2) to 6,702,434 (BJ2), with an average of 3,379207 . The information for each of 39 accession reads was aligned to the simulated reference genome of BQ2, which has the highest tag number $(5,192,250)$ (Table S1). In the 39 Bupleurum samples, an average of 2,657,398 (78.1\%) reads were mapped to the reference genome. Among them, BJ3 showed the highest mapping rate (87.97\%), and DY2 showed the lowest mapping rate (72.16\%) (Table S2). After successful mapping, a total of 85,251 GBS SNPs were recognized, and 83,898 SNPs were obtained after filtering out multiple allele loci using Beagle software.

\subsection{Population structure analysis}

The K-value was calculated to evaluate the different clusters of these 39 Bupleurum samples based on 83,898 genome-wide SNPs with high-quality data. The cross-validation (CV) error was estimated to test the optimal K-value. From 2 to 11 , the best K-value showed four groups with the highest probability of clustering according to the genetic background information instead of the sample collection location (Fig. 1). The four groups of 39 accessions accounted for $30.77 \%, 28.21 \%, 25.64 \%$ and $15.38 \%$, respectively. Cluster $\otimes$ contained 12 accessions of $B$. chinense, mainly from Hebei Province and Beijing, except for one sample from Heilongjiang Province (HB1, HB2, HB3, HB4, HB5, HB6, BJ2, BJ3, BJ4, BJ5, BJ7, MH1). Cluster $\mathbb{2}$ included 11 accessions of $B$. chinense mainly from Shaanxi, Heilongjiang and Gansu Provinces (HRB1, HRB2, HRB3, DF4, DF5, DF6, GS1, GS2, GS3, GS4, GS6). Cluster $₫$ included all 10 resources from Heilongjiang Province. However, these samples belong to different species. Three accessions were $B$. chinense (BQ1, BQ3, ZZ1), 3 samples were $B$. sibiricum Vest. (Q1, Q2, Q3), 3 accessions were B. longiradiatum Turcz. (DY1, DY2, DY3) and one sample was B. scorzonerifolium Willd. $(\mathrm{MH} 3)$. Cluster $₫$ contained the remaining 6 B. chinense resources from Heilongjiang Province $(Z Z 2, Z Z 3, D Q 1, D Q 2, D Q 3$, $\mathrm{BQ} 2)$.

Neighbour-joining (NJ) tree analysis was performed based on 83,898 SNPs and classified the 39 Bupleurum into three major groups: (1) B. chinense samples from Shaanxi Province; (2) B. chinense accessions from Gansu, Hebei Province and Beijing; and (3) B. chinense, $B$. scorzonerifolium, B. sibiricum and B. longiradiatum resources from Heilongjiang Province. Group $\otimes$ was split into 2 subgroups on the basis of the population structure results. The first subgroup mainly corresponded 
to samples from Gansu Province, and the second subgroup corresponded to samples from Hebei Province and the city of Beijing (Fig. 2).

The relationship between the 39 Bupleurum accessions was explored based on the broad geographical regions using PCA. The samples showed a relatively tight distribution in the PCA results (Fig. 3). The majority of the $B$. chinense accessions

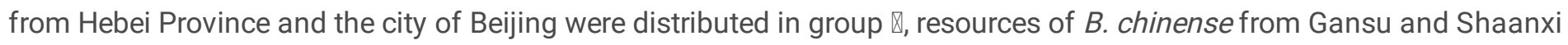
Provinces were clustered in group $\Downarrow$. Group $\$ contained the other three "Chai-hu" species from Heilongjiang Province, and

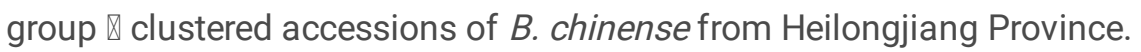

\subsection{Genetic diversity analysis}

The tested Bupleurum materials were divided into 11 populations according to geographical origin and species (Table 1). The observed heterozygosity $(\mathrm{Ho})$ ranged from 0.21 to 0.27 with an average of 0.24 . The expected heterozygosity $(\mathrm{He})$ value ranged from 0.15 to 0.19 with an average of 0.17 . The polymorphic loci number (PLN) ranged from 30,724 to 45,778 , and the average was 37,358 . The percentage of polymorphic loci (PPL) ranged from 38.97 to 58.07 , and the average PPL was 47.38. The mean heterozygosity, Ho, was greater than the expected heterozygosity, He, indicating that the genetic diversity of Bupleurum was narrow (Table 2).

Table 2

Genetic diversity of Bupleurum from different geographical resources and species

\begin{tabular}{|c|c|c|c|c|c|c|}
\hline Origin & Species & Number & Ho & $\mathrm{He}$ & PLN & PPL/\% \\
\hline Hebei & \multirow[t]{8}{*}{ B. chinense DC. } & 6 & 0.2427 & 0.1718 & 41196 & 52.2533 \\
\hline Beijing & & 5 & 0.2082 & 0.1731 & 43285 & 54.903 \\
\hline Gansu & & 5 & 0.2419 & 0.1862 & 45778 & 58.0652 \\
\hline Harbin & & 3 & 0.2485 & 0.1774 & 38116 & 48.3466 \\
\hline Daqing & & 3 & 0.2452 & 0.1766 & 38409 & 48.7183 \\
\hline Zhaozhou & & 3 & 0.2157 & 0.1538 & 32949 & 41.7928 \\
\hline Qiqihar & & 3 & 0.2701 & 0.1919 & 41160 & 52.2077 \\
\hline Shaanxi & & 3 & 0.2262 & 0.1566 & 33302 & 42.2405 \\
\hline Heilongjiang & B. scorzonerifolium Willd. & 2 & 0.2359 & 0.1599 & 30724 & 38.9706 \\
\hline Heilongjiang & B. sibiricum Vest. & 3 & 0.2299 & 0.157 & 32587 & 41.3336 \\
\hline Heilongjiang & B. longiradiatum Turcz. & 3 & 0.2719 & 0.1711 & 33439 & 42.4143 \\
\hline Average & & & 0.24 & 0.17 & 37358.63 & 47.38 \\
\hline
\end{tabular}

The genetic distance (GD) value between 11 populations from different geographical resources and species was -0.3916 to 0.2845 , indicating that the genetic distance between populations was small and that the genetic basis was relatively narrow. Among these different species, $B$. scorzonerifolium Willd. showed the maximum genetic distance from $B$. chinense DC. from Heilongjiang Province (0.068), indicating that these resources possess different genetic background information but have the same effect as the Chinese traditional medicine "Chai-hu" (Table 3). The nucleotide diversity analysis of each population found that the mean nucleotide diversity $(\pi)$ of the total loci was 0.2019 , indicating that polymorphisms within populations were relatively rare. The AMOVA results revealed that the genetic variation within populations (23.36\%) was greater than that among populations (2.46\%), indicating that relatively high gene information exchange occurred within populations and low differentiation occurred among populations (Table 4). 
Table 3

Genetic distance matrix of Bupleurum samples from different geographical and species resources

\begin{tabular}{|c|c|c|c|c|c|c|c|c|c|c|c|}
\hline & HB & BJ & GS & BQ & HRB & DQ & $\mathrm{MH}$ & ZZ & $\mathbf{Q}$ & DY & DF \\
\hline $\mathrm{HB}$ & 0 & & & & & & & & & & \\
\hline BJ & 0.0821 & 0 & & & & & & & & & \\
\hline GS & 0.2138 & 0.1201 & 0 & & & & & & & & \\
\hline $\mathrm{BQ}$ & 0.1817 & 0.0212 & 0.1724 & 0 & & & & & & & \\
\hline HRB & 0.2854 & 0.1422 & 0.2380 & 0.1409 & 0 & & & & & & \\
\hline DQ & 0.1899 & 0.1932 & 0.1028 & 0.2958 & 0.1436 & 0 & & & & & \\
\hline $\mathrm{MH}$ & 0.4505 & 0.2377 & 0.1085 & 0.6804 & 0.3124 & 0.3964 & 0 & & & & \\
\hline $\mathrm{ZZ}$ & 0.1506 & 0.2650 & 0.0173 & 0.2029 & 0.2369 & 0.4547 & 0.4427 & 0 & & & \\
\hline $\mathrm{Q}$ & 0.4181 & 0.2036 & 0.0861 & 0.3977 & 0.0866 & 0.3710 & 0.5262 & 0.4284 & 0 & & \\
\hline DY & 0.3040 & 0.0640 & 0.1581 & 0.2257 & 0.0289 & 0.1311 & 0.3916 & 0.2587 & 0.4742 & 0 & \\
\hline DF & 0.2559 & 0.1151 & 0.2566 & 0.2600 & 0.0259 & 0.4367 & 0.3370 & 0.2132 & 0.5405 & 0.4465 & 0 \\
\hline
\end{tabular}

Table 4

Analysis of molecular variance (AMOVA) for 39 Bupleurum accessions

\begin{tabular}{|lllll|}
\hline Source of variation & df & Sum of squares & Variance components & Percentage of variation \\
\hline Among populations & 10 & 71131.032 & $191.35821 \mathrm{Va}$ & 2.46 \\
\hline $\begin{array}{l}\text { Among individuals } \\
\text { within populations }\end{array}$ & 28 & 161578.25 & $-1815.00744 \mathrm{Vb}$ & -23.34 \\
\hline Within individuals & 39 & 366626 & $9400.66667 \mathrm{Vc}$ & 120.88 \\
\hline Total & 77 & 599335.282 & 7777.01743 & \\
\hline
\end{tabular}

\begin{tabular}{|lllllll|}
\hline ZZ2 & 2603018 & 1741851 & $66.92 \%$ & 27.39 & $28.65 \%$ & $17.28 \%$ \\
\hline$Z Z 3$ & 4030106 & 3343969 & $82.97 \%$ & 40.52 & $36.55 \%$ & $22.55 \%$ \\
\hline
\end{tabular}

\section{Discussion}

The traditional herb "Chai-hu" is a medicinal plant-based remedy used worldwide that contains the roots of several species from the genus Bupleurum L. according to the WHO in 1999 [28] and shows uneven quality among these different resources in China [29]. It is essential to estimate the diversity and relationships among "Chai-hu" resources to identify excellent breeding materials. Previous research on the population structure and genetic diversity of "Chai-hu" has been carried out based on molecular markers including RAPD, AFLP, ISSR and ITS sequences [30-32]. SNP markers are commonly applied because of their low cost, high throughput and high abundance. Although there is a lack of studies on the thorough collection of Chai-hu accessions in China, the population structure and genetic diversity of 39 Bupleurum accessions from 11 different areas are reported with 83,898 SNPs detected by GBS analysis in this work.

Medicinal Bupleurum is widely distributed in all regions of China except Hainan Island; clearly, Bupleurum has displayed a wide genetic diversity over a long period of domestication to adapt to diverse environments. Bupleurum resources can be divided into different groups according to their traditional uses. Using amplified fragment length polymorphisms, 11 
Bupleurum strains were classified into 3 different subsets, among which 2 varieties of Bupleurum chinense DC. From Henan and Hebei Provinces showed the closest relationship [31]. ISSR markers were used to analyse the genetic distance of 11 Bupleurum chinense samples, indicating that the genetic variation has a certain relation with geographic distribution [33]. In recent decades, seeds of $B$. chinense DC. resources grown in several provinces or areas in China have mainly originated from Gansu and Shaanxi Provinces; this phenomenon is strongly supported by genetic diversity results with genetic distances ranging from $0.0173 \otimes 0.4547$, which suggested low genetic diversity within $B$. chinense DC. samples. The nearest accessions were from Heilongjiang and Gansu Provinces, indicating that cultivated samples in Heilongjiang Province were introduced from Gansu Province, which is consistent with the present situation regarding the origin of seeds on the medicinal market in China.

Species grouping of Bupleurum strains was also demonstrated with PCA. Accessions of $B$. sibiricum Vest. (Q1, Q2, Q3), 3 accessions of $B$. longiradiatum Turcz. (DY1, DY2, DY3) and 2 samples of $B$. scorzonerifolium Willd. (MH1, MH3) were clearly distinguished from accessions of $B$. chinense DC., emphasizing the trouble with traditional discrimination methods based on phenotypes because of the substantial divergence among these different species. However, the relationship between these different species was also relatively close, ranging from $0.0289 \varangle 0.6804$, suggesting that they may have evolved from a common genetic origin with a lack of diversity at the DNA level. The data collected in this research will be helpful for the establishment of quality criteria for "Chai-hu" and for the formulation of reasonable clinical use strategies. At the same time, the utilization of SNP markers could not only be applied in resource discrimination but also offer genetic information for screening and molecular breeding of medicinal plant varieties.

\section{Declarations}

Acknowledgements This work was supported by the Key project at central government level: The ability establishment of sustainable use for valuable Chinese medicine resources (2060302) and the State Key Laboratory of Pharmaceutical Newtech for Chinese Medicine (SKL2020M0302).

Author contributions M J wrote the manuscript, and checked and analyzed data. S Y, W-C R, and N-N X collected data and revised the manuscript. $\mathrm{H}-\mathrm{Y} \mathrm{L}, \mathrm{M}-\mathrm{Q} Z \mathrm{Z}$ and $\mathrm{M}-\mathrm{Q} \mathrm{L}$ helped revised the manuscript. W M critically designed the experiment.

Conflict of interest The authors declare that they have no conflicts of interest.

Ethical approval This aitical does not contain any experiment with human participants or animals.

\section{References}

1. Feng YJ, Wu ZW, Luo YY et al (2019) A new triterpene diglycoside from the roots of Bupleurum chinense DC. and its inhibitory effect on adipogensis in 3T3-L1 cells[J]. Med Chem Res 28:239-245

2. Pan SL (2006) Bupleurum species: scientific evaluation and clinical applications. CRC Press, Boca Raton

3. Law YK, Mo JF, Wong KW (2014) Autophagic effects of Chaihu (dried roots of Bupleurum Chinense DC or Bupleurum scorzoneraefolium WILD) [J]. Chin Med 9:21

4. Yu J, Deng A, Wu L et al (2013) Osteoclast-inhibiting saikosaponin derivatives from Bupleurum Chinense[J]. Fitoterapia 85:101-108

5. Li DQ, Wu J, Liu LY et al (2015) Cytotoxic triterpenoid glycosides (saikosaponins) from the roots of Bupleurum chinense[J], vol 25. Bioorganic \& Medicinal Chemistry Letters, pp 3887-3892

6. Li HY, Zhao YH, Zeng MJ et al (2017) Saikosaponin D relieves unpredictable chronic mild stress induced depressive-like behavior in rats: involvement of HPA axis and hippocampal neurogenesis[J]. Psychopharmacology 234:3385-3394

7. Wang Y, Qiang G, Cheng Z et al (2017) New saikosaponins from the roots of Bupleurum chinense[J]. Phytochem Lett 21:183-189

Page $8 / 12$ 
8. Wang HW, Liu M, Zhong TD et al (2015) Saikosaponin-d attenuates ventilator-induced lung injury in rats[J]. Int J Clin Exp Med 8:15137-15145

9. Chen XQ, Chen SJ, Liang WN et al (2018) Saikosaponin A attenuates perimenopausal depression-like symptoms by chronic unpredictable mild stress[J]. Neurosci Lett 662:283-289

10. Lorrai I, Maccioni P, Carai M et al (2017) Suppressing effect of saikosaponin $A$, an active ingredient of Bupleurum falcatum, on chocolate self-administration and reinstatement of chocolate seeking in rats[J]. Neurosci Lett 638:211217

11. National Pharmacopoeia Committee. Pharmacopoeia of Peoples Republic of China [M]. Part 1. Beijing: Chemical Industry Press (2020): Appendix 2: 293

12. Sui C, He WJ, Lin CS et al (2009) Development of genomic SSR and potential EST-SSR markers in Bupleurum chinense DC.[J]. Afr J Biotechnol 8:6233-6240

13. Zhao X, Liu C, Xue W et al (2015) ISSR Research on Germplasm of Bupleurum chinense DC. in Beijing[J]. Modern Chinese Medicine 10:1008-1013

14. Yang W, Bai Y, Ji-Ying HU (2013) ISSR Research on Germplasm of Bupleurum chinense DC. in Baokang[J]. Research and Practice on Chinese Medicines, 2:25-27

15. Lee KJ, Lee JR, Sebasti NR et al (2019) Genetic Diversity Assessed by Genotyping by Sequencing (GBS) in Watermelon Germplasm[J]. Genes 10:822-834

16. Fu YB, Peterson GW (2011) Genetic Diversity Analysis with 454 Pyrosequencing and Genomic Reduction Confirmed the Eastern and Western Division in the Cultivated Barley Gene Pool[J]. Plant Genome 4:226-237

17. Peterson BK, Weber JN, Kay EH et al (2012) Double Digest RADseq: An Inexpensive Method for De Novo SNP Discovery and Genotyping in Model and Non-Model Species[J]. PLoS ONE 7:e37135

18. Peterson G, Dong Y, Horbach C et al (2014) Genotyping-By-Sequencing for Plant Genetic Diversity Analysis: A Lab Guide for SNP Genotyping[J]. Diversity 6:665-680

19. Yu D, Wang H, Gu W et al (2021) Genetic diversity and population structure of popcorn germplasm resources using genome-wide SNPs through genotyping-by-sequencing[J]. Genetic Resources and Crop Evolution, pp 1-11

20. Lee HY, Jang S, Yu CR et al (2020) Population Structure and Genetic Diversity of Cucurbita moschata Based on Genome-Wide High-Quality SNPs[J]. Plants 10:56-65

21. Yang X, Tan B, Liu H et al (2020) Genetic Diversity and Population Structure of Asian and European Common Wheat Accessions Based on Genotyping-By-Sequencing[J].Frontiers in Genetics, 1-11

22. Li H, Durbin R Fast and accurate short read alignment with Burrows-Wheeler transform. Bioinformatics,2009a, 25:17541760

23. Li H, Handsaker B, Wysoker A et al The Sequence Alignment/Map format and SAMtools. Bioinformatics,2009b, 25:2078-2079

24. Wang K, Li M, Hakonarson H (2010) ANNOVAR: functional annotation of genetic variants from high-throughput sequencing data. Nucleic Acids Res 38:e164

25. Alexander DH, Novembre J, Lange K (2009) Fast model-based estimation of ancestry in nrelated individuals. Genome, Res 19:1655-1664

26. Excoffier L, Laval G, Schneider S (2005) Arlequin (version 3.0): an integrated software package for population genetics data analysis[J]. Evol Bioinform Online 1:47-50

27. Saitou N, Nei M (1987) The neighbor-joining method: a new method for reconstructing phylogenetic trees.[J]. Molecular Biology \& Evolution, (4):406

28. World Health Organization (1999) WHO Monographs on Selected Medicinal Plants[M], vol 1. World Health Organization, Geneva 
29. Huang HQ, Zhang X, Xu ZX et al (2009) Fast determination of saikosaponins in Bupleurum by rapid resolution liquid chromatography with evaporative light scattering detection[J]. J Pharm Biomed Anal 49(4):1048-1055

30. Huang W, Sun P, Zhang WS et al (2008) Genetic Diversity of Bupleurum chinense DC. populations from different altitudes in Dongling mountain district in Beijing[J]. Plant Genetic Resources 9(4):453-457

31. Ke SY, Shi LL, Ma YZ et al (2015) Evaluation of the genetic diversity of Bupleurum using amplified fragment length polymorphism analysis[J], vol 14. GENET MOL RES, pp 2590-25991

32. Du SM, Wang G, Liu YM et al (2013) Study on Biological Materials with Genetic Diversity of Bupleurum marginatum in Northwest of Hubei Province of China Base on ISSR[J]. Adv Mater Res 830:463-468

33. Li YH, Yu XL, Ou XJ et al (2018) Genetic Diversity of Different Origin Bupleurum chinese Detected by ISSR Analysis[J]. Lishizhen Medicine and Materia Medica Research 29:1728-1731

\section{Figures}

$\left.\begin{array}{l}\circ- \\ \vdots \\ 0 \\ \vdots \\ \vdots \\ \vdots \\ \vdots \\ \vdots \\ \vdots \\ \vdots\end{array}\right]$

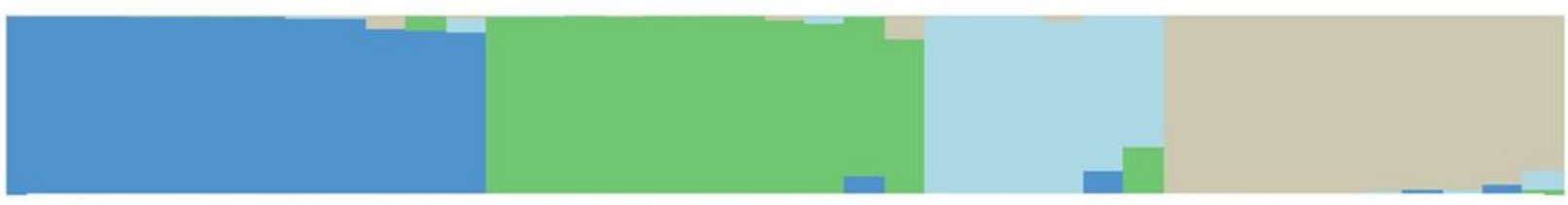

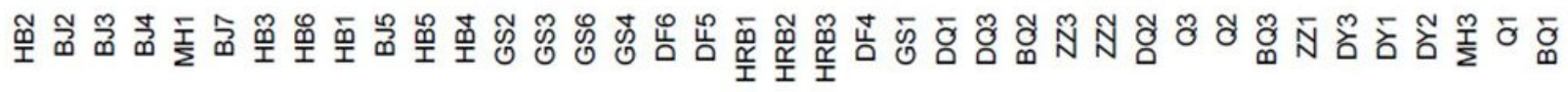

Figure 1

Population structure of 39 genotypes with $\mathrm{K}=4$. Each genotype is represented by a single vertical line 


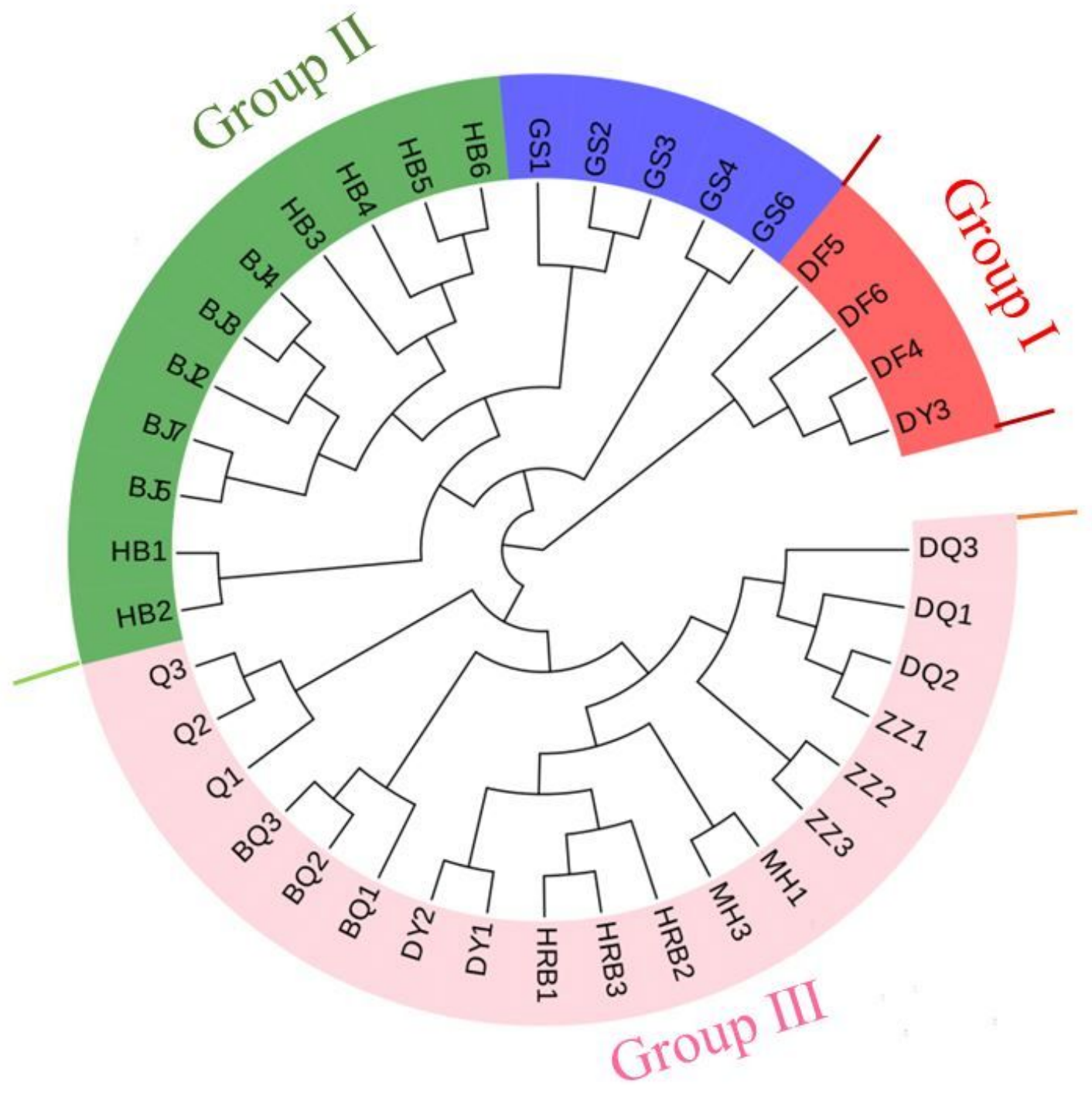

Figure 2

Neighbour-joining $(\mathrm{NJ})$ tree analysis of 39 Bupleurum accessions based on population structure 

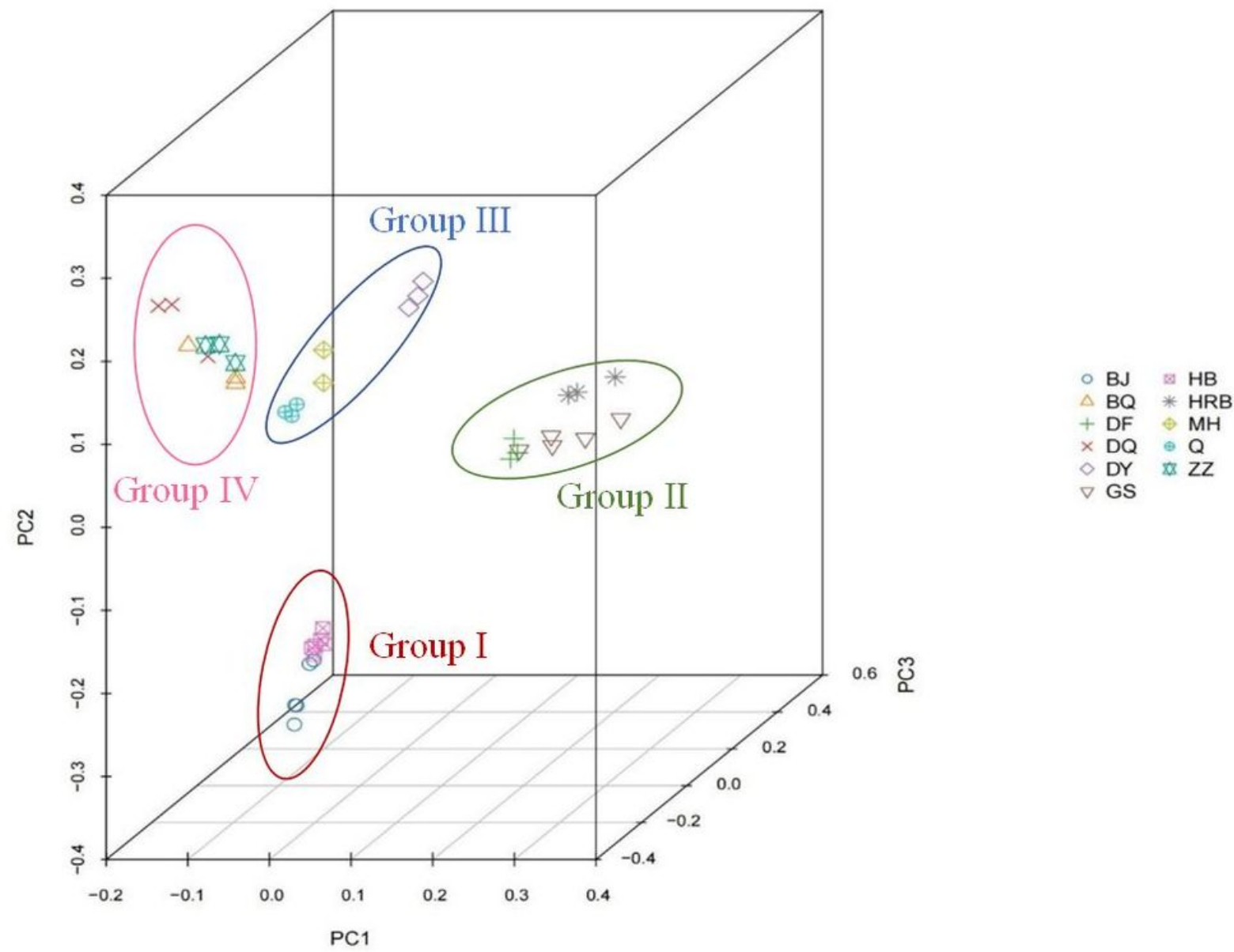

\section{Figure 3}

PCA (principal component analysis) based on SNP information with genetic distances among 39 Bupleurum accessions

\section{Supplementary Files}

This is a list of supplementary files associated with this preprint. Click to download.

- JiangmingMBRsupplementarytable.docx 